\title{
Index to Volume 35, 2019
}

\author{
Contents of Volume 35, Numbers 1-4 \\ Articles, See Author Index \\ Editorial Collaborators 913 \\ Index 919 \\ In Memoriam 285 \\ Letter to Editor 1 \\ Preface 699
}

\section{Author Index}

\begin{abstract}
Abraham, K.G. and Amaya, A. Probing for Informal Work Activity $487-508$

Amaya, A., See Abraham, K.G.

Augustin, T., See Endres, E.

Bates, N., Y.A. García Trejo, and M. Vines. Are Sexual Minorities Hard-to-Survey? Insights from

the 2020 Census Barriers, Attitudes, and Motivators Study (CBAMS) Survey . . . . . . . . . . . . . . . .709-729

Bates, N., S. Steinmetz, and M. Fischer. Preface. . . . . . . . . . . . . . . . . . . . . . . . . . . . . . . .699-707

Beręsewicz, M. Correlates of Representation Errors in Internet Data Sources for Real Estate Market . . . . .509-529

Białek, J. Remarks on Geo-Logarithmic Price Indices . . . . . . . . . . . . . . . . . . . . . . . . . . . . . 287-317

Bikker, R., Van den Brakel, J., Krieg, S., Ouwehand, P., and Van der Stegen, R. Consistent

Multivariate Seasonal Adjustment for Gross Domestic Product and its Breakdown in Expenditures. . . . . . . 9-30

Blom, A.G., See Sakshaug, J.W.

Call, K., See Pascale, J.

Chakraborty, R., Kavonius, I.K., Pérez-Duarte, S., and Vermeulen, P. Is the Top Tail of the

Wealth Distribution the Missing Link between the Household Finance and Consumption Survey and

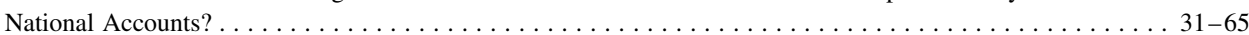

Chipperfield, J., Newman, J., Thompson, G., Ma, Y., and Lin, Y-X. Prospects for Protecting Business

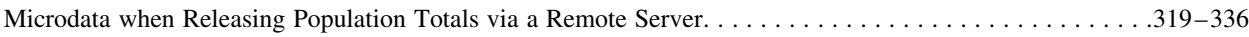

Dahlhamer, J.M., Galinsky, A.M., and Joestl, S.S. Asking about Sexual Identity on the National

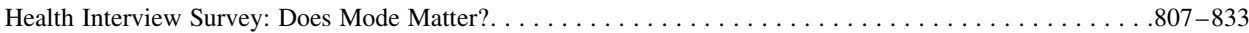

Dalla Chiara, E., Menon, M., and Perali, F. An Integrated Database to Measure Living Standards. . . . . . .531-576

D’Aurizio, L. and Papadia, G. Using Administrative Data to Evaluate Sampling Bias in a Business
\end{abstract}

Panel Survey. . . . . . . . . . . . . . . . . . . . . . . . . . . . . . . . . . . 67-92

Dennis, J.M., See Michaels, S.

De Wolf, P-P., Van der Laan, J., and Zult, D. Connecting Correction Methods for Linkage Error

in Capture-Recapture. . . . . . . . . . . . . . . . . . . . . . . . . . . . . . . . . . . . . . . . 577-597

Dinkelmann, K., Granda, P., and Shove, M. Enhancing Survey Quality: Continuous

Data Processing Systems . . . . . . . . . . . . . . . . . . . . . . . . . . . . . . . . . . . . . 337-352

Drechsler, J., Kiesl, H., Meinfelder, F., Raghunathan, T.E., Rubin, D.B., Schenker, N., and Zell,

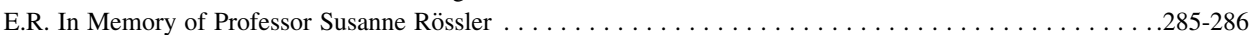

Dykema, J., Garbarski, D., Wall, I.F., and Farrar Edwards, D. Measuring Trust in Medical

Researchers: Adding Insights from Cognitive Interviews to Examine Agree-Disagree and

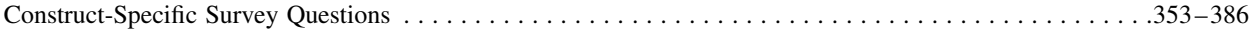

Edgar, J., See Holzberg, J.

Eggleston, J. Item Response Rates for Composite Variables . . . . . . . . . . . . . . . . . . . . . . . . . . 387-408

Ellis, R., See Holzberg, J.

Endres, E., Fink, P., and Augustin, T. Imprecise Imputation: A Nonparametric Micro Approach

Reflecting the Natural Uncertainty of Statistical Matching with Categorical Data. . . 
Farrar Edwards, D., See Dykema, J.,

Felderer, B., Kirchner, A., and Kreuter, F. The Effect of Survey Mode on Data Quality: Disentangling

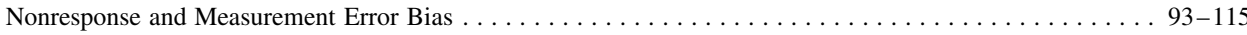

Fertig, A., See Pascale, J.

Fink, P., See Endres, E.

Fischer, M., See Bates, N.

Fischer, M., See Steinmetz, S.

Galinsky, A.M., See Dahlhamer, J.M.

Ganesh, N., See Michaels, S.

Garbarski, D., See Dykema, J.

García Trejo, Y.A., See Bates, N.

Georgiou, A.V. Letter to the Editor: Extracting Statistical Offices from Policy-Making Bodies to

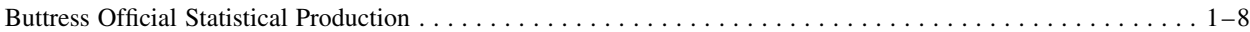

Gilbert, T., See Truman, J.L.

Granda, P., See Dinkelmann, K.

Holmberg, A., See Lothian, J.

Holzberg, J., Ellis, R., Kaplan, R., Virgile, M., and Edgar, J. Can They and Will They? Exploring

Proxy Response of Sexual Orientation and Gender Identity in the Current Population Survey . . . . . . . . . 885-911

Joestl, S.S., See Dahlhamer, J.M.

Johansen, I., See Zhang, L-C.

Kaplan, R., See Holzberg, J.

Kavonius, I.K., See Chakraborty, R.

Keogh, G. A Lexical. Approach to Estimating Environmental Goods and Services Output in the

Construction Sector via Soft Classification of Enterprise Activity Descriptions

Using Latent Dirichlet Allocation. . . . . . . . . . . . . . . . . . . . . . . . . . . . . . . . . . 625-651

Kiesl, H., See Drechsler, J.

Kirchner, A., See Felderer, B.

Koskinen, J., See Pina-Sánchez, J.

Kreuter, F., See Felderer, B.

Krieg, S., See Bikker, R.

Kroh, M., See Kühne, S.

Kühne, S., Kroh, M., and Richter, D. Comparing Self-Reported and Partnership-Inferred Sexual

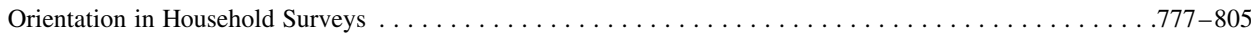

Liao, P-S., Saris, W.E., and Zavala-Rojas, D. Cross-National Comparison of Equivalence and

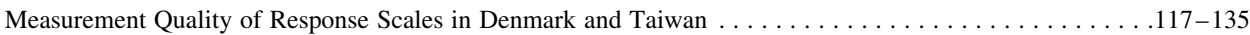

Lin, Y-X., See Chipperfield, J.

Lothian, J., Holmberg, A., and Seyb, A. An Evolutionary Schema for Using "it-is-what-it-is"

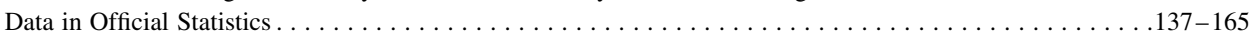

Ma, Y., See Chipperfield, J.

Maples, J.J., See O’Hara, B.

Massing, M., Wasmer, M., Wolf, C., and Zuell, C. How Standardized is Occupational Coding?

A Comparison of Results from Different Coding Agencies in Germany . . . . . . . . . . . . . . . . . . . . .167-187

Medalia, C., See O'Hara, B.

Meinfelder, F., Drechsler, J.

Menon, M., See Dalla Chiara, E.

Michaels, S., Pineau, V., Reimer, B., Ganesh, N., Dennis, J.M. Test of a Hybrid Method of

Sampling the LGBT Population: Web Respondent Driven Sampling with Seeds from a

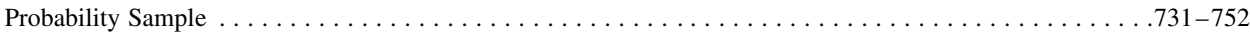

Mishel, M. Intersections between Sexual Identity, Sexual Attraction, and Sexual Behavior

among a Nationally Representative Sample of American Men and Women . . . . . . . . . . . . . . . . . . .859-884

Morgan, R.E., See Truman, J.L.

Newman, J., See Chipperfield, J.

Nygaard, R., See Zhang, L-C.

O’Hara, B., Medalia, C., and Maples, J.J. Modeling a Bridge When Survey Questions Change:

Evidence from the Current Population Survey Health Insurance Redesign . . . . . . . . . . . . . . . . . . . . .189-202

Ouwehand, P., See Bikker, R.

Papadia, G., See D’Aurizio, L. 
Pascale, J., Fertig, A., and Call, K. Validation of Two Federal Health Insurance Survey Modules

After Affordable Care Act Implementation . . . . . . . . . . . . . . . . . . . . . . . . . . . . . .409-460

Perali, F., See Dalla Chiara, E.

Pérez-Duarte, S., See Chakraborty, R.

Perez Ruiz, D.A., See Sakshaug, J.W.

Pina-Sánchez, J., Koskinen, J., and Plewis, I. Adjusting for Measurement Error in Retrospectively

Reported Work Histories: An Analysis Using Swedish Register Data.

Pineau, V., See Michaels, S.

Plewis, I., See Pina-Sánchez, J.

Raghunathan, T.E., See Drechsler, J.

Reimer, B., See Michaels, S.

Richter, D., See Kühne, S.

Rubin, D.B., See Drechsler, J.

Sakshaug, J.W., Wiśniowski, A., Perez Ruiz, D.A., and Blom, A.G. Supplementing Small Probability

Samples with Nonprobability Samples: A Bayesian Approach . . . . . . . . . . . . . . . . . . 653-681

Saris, W.E., See Liao, P-S.

Schenker, N., See Drechsler, J.

Seyb, A., See Lothian, J.

Shove, M., See Dinkelmann, K.

Steinmetz, S., See Bates, N.

Steinmetz, S. and Fischer, M. Surveying Persons in Same-Sex Relationships in a Probabilistic Way - An

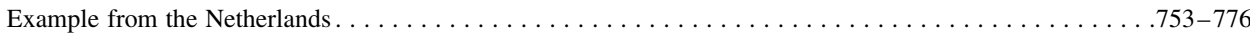

Tarnow-Mordi, R.C., See Webster, M.

Thompson, G., See Chipperfield, J.

Truman, J.L., Morgan, R.E., Gilbert, T., and Vaghela, P. Measuring Sexual Orientation and Gender

Identity in the National Crime Victimization Survey

Vaghela, P., See Truman, J.L.

Van den Brakel, J., See Bikker, R.

Van der Laan, J., See De Wolf, P-P.

Van der Stegen, R., See Bikker, R.

Vermeulen, P., See Chakraborty, R.

Virgile, M., See Holzberg, J.

Wall, I.F., See Dykema, J.

Wasmer, M., See Massing, M.

Webster, M. and Tarnow-Mordi, R.C. Decomposing Multilateral Price Indexes into the

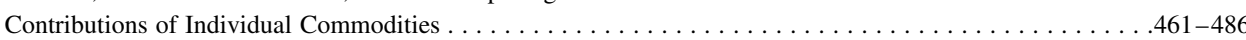

Willekens, F. Evidence-Based Monitoring of International Migration Flows in Europe . . . . . . . . . . . . .231-277

Vines, M., See Bates, N.

Wiśniowski, A., See Sakshaug, J.W.

Wolf, C., See Massing, M.

Zavala-Rojas, D., See Liao, P-S.

Zell, E.R., See Drechsler, J.

Zhang, L-C. A Note on Dual System Population Size Estimator . . . . . . . . . . . . . . . . . . . . . . . . .279-283

Zhang, L-C., Johansen, I., and Nygaard, R. Tests for Price Indices in a Dynamic Item Universe . . . . . . . 683-697

Zuell, C., See Massing, M.

Zult, D., See De Wolf, P-P. 